\title{
PREGNANCY IN A NON-COMMUNICATING RUDIMENTARY HORN OF A UNICORNUATE UTERUS: A CASE REPORT
}

\author{
B. Anuradha ${ }^{1}$, B. Sudharani², K. Sambasiva ${ }^{3}$, V. Sarath Chand ${ }^{4}$
}

\section{HOW TO CITE THIS ARTICLE:}

B. Anuradha, B. Sudharani, K. Sambasiva, V. Sarath Chand. "Pregnancy in a Non-Communicating Rudimentary Horn of a Unicornuate Uterus: A Case Report". Journal of Evolution of Medical and Dental Sciences 2015; Vol. 4, Issue 66, August 17; Page: 11580-11585, DOI: 10.14260/jemds/2015/1672

INTRODUCTION: Pregnancy in a rudimentary horn of a unicornuate uterus is rare. ${ }^{1}$ An incidence of 1 in 76,000-150,000 pregnancies is reported in the literature. ${ }^{2,3}$ We present a case report of rupture of a 25 weeks pregnancy in the non-communicating rudimentary horn of a unicornuate uterus. Congenital malformations of the uterus, also known as Mullerian duct anomalies, are rare in general population (Approx. 1\%). These abnormalities result from arrested development, abnormal formation or incomplete fusion of mesonephric ducts. Unicornuate uterus results from unilateral arrested mullerian duct development. Rarely unicornuate uterus may also have a rudimentary horn, more on the right than on the left side. The incidence of unicornuate uterus is estimated to be 1:250 and its occurrence with rudimentary horn is 1:100,000. Such anomalies are reported to result in increased rate of miscarriages, recurrent pregnancy losses, preterm labor, infertility and other obstetric complications. Conception in rudimentary horn arises either from a small communication with the uterine cavity (Communicating) or by Trans peritoneal migration of the fertilized ovum from the contra-lateral side (Non-communicating). The proportion of non-communicating rudimentary horns is $70-90 \%$. The frequency of pregnancy in rudimentary horn is reported to be 1:76000. The clinical presentations vary from being asymptomatic to vague complaints of mild lower abdominal pain with gastrointestinal upset to its severest form of acute abdomen with hemorrhagic shock. The most significant threat of a rudimentary horn pregnancy is the risk of rupture because of poorly developed musculature. In view of the paucity of literature on rare observation of pregnancy in rudimentary horn of uterus, the case reported here is of crucial importance.

KEYWORDS: Unicornuate uterus, Rudimentary horn, Secondary abdominal pregnancy.

\section{CASE HISTORY:}

- 20 yrs Gravida 2 para 1 female with 25weeks of gestation according to her LMP referred to radiology department for obstetric scan, as she had complained of decreased fetal movements and abdominal pain since two days. She did not underwent ultrasound examination previously in the present pregnancy.

- She had previous history of full term normal vaginal delivery of live child 2 years back. Her post-partum period was uneventful.

- Her past medical and gynecologic histories were unremarkable with regular menses and without dysmenorrhea.

- Her vital parameters were normal. Her abdominal examination revealed suprapubic mass corresponds to 24 to 26 weeks of gestation.

Per vaginal examination shows fullness in the left fornix. 


\section{IMAGING FEATURES:}

- Patient was sent for ultrasound examination which showed a dead intra-abdominal fetus of 25-26 weeks of gestation with scanty amniotic fluid, with empty uterus lying in the pelvis towards left side and minimal free fluid in the peritoneal cavity (Fig. 1). MRI was also done, MRI had showed a fetus outside the uterus in the abdominal cavity within a clearly defined gestational sac. (Fig. 2).

- The placenta was seen with definitive borders located in the antero inferior part of the sac.

- No signs of placental invasion of the neighboring structures were observed.

- Ruptured rudimentary horn seen with fetus lying in abdominal cavity. No cavity connection between the gestational horn and the uterus (Fig. 4, 5).

Uterus noted towards left side of pelvis. Ruptured rudimentary horn with placenta noted on right side (Fig. 4).

Fig. (1): Showing Ultrasonographic evaluation revealed bulky uterus (red arrow) displaced to left side by the neighboring gestational sac (block arrow) with endometrial collection and minimal free fluid.

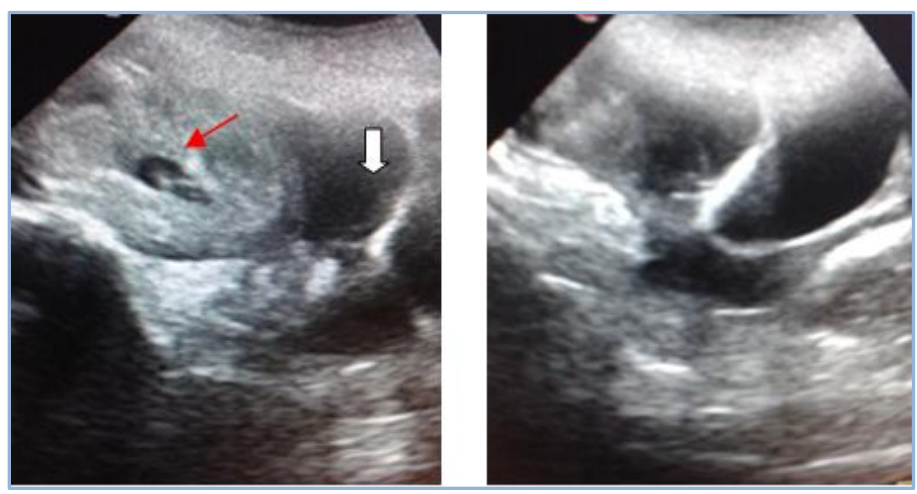

\section{Figure 1}

Fig. (2): Showing Coronal T2WI images showing fetus (Red arrow) lying in the abdominal cavity with surrounding gestation sac. Placenta (Blue arrow) seen anteroinferiorly in the pelvis.

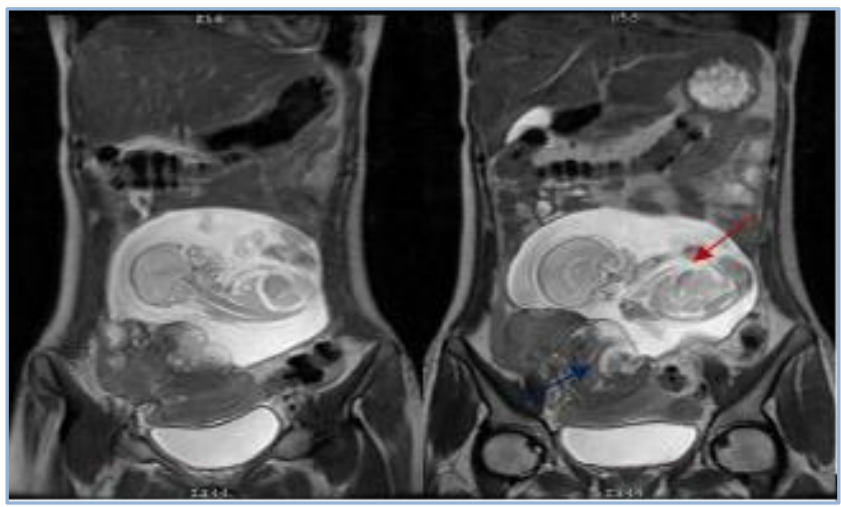

\section{Figure 2}




\section{CASE REPORT}

Fig. (3): CorT2WI images posteriorly showing rudimentary horn (Red arrow) towards right side of pelvis and uterus (block arrow) pushed towards left.
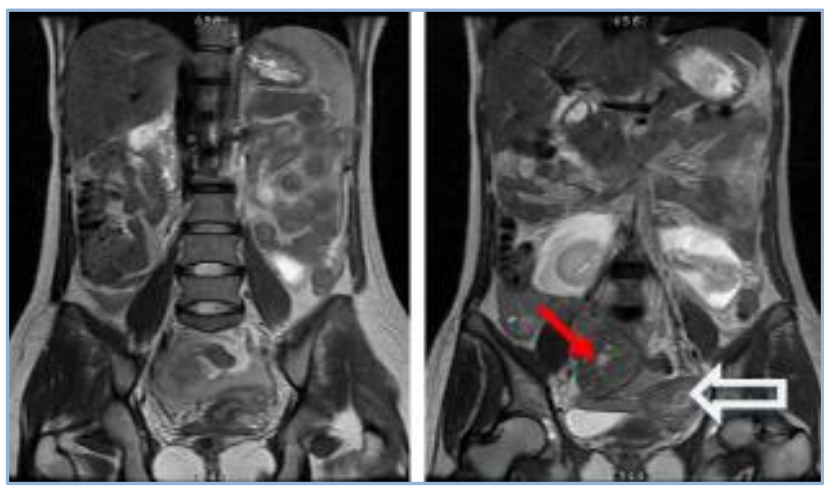

Figure 3

Fig. (4): Axial T2WI images showing fetus in transverse lie (Red arrow), in next sections cord, placental attachment (Orange arrow) and ruptured horn (blue arrow).

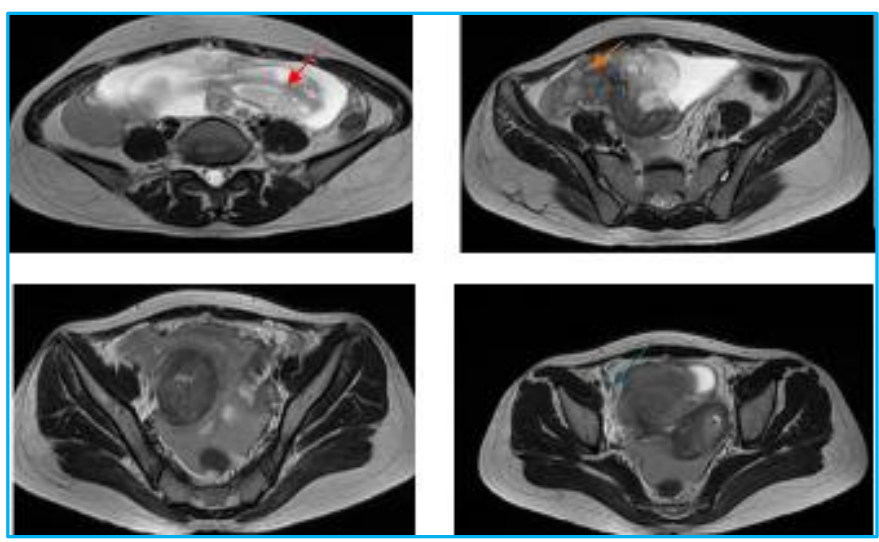

Figure 4

Fig. (5): Showing Sag T2WI images from right to left shows ruptured horn (red arrow) and normal uterus (blue arrow).

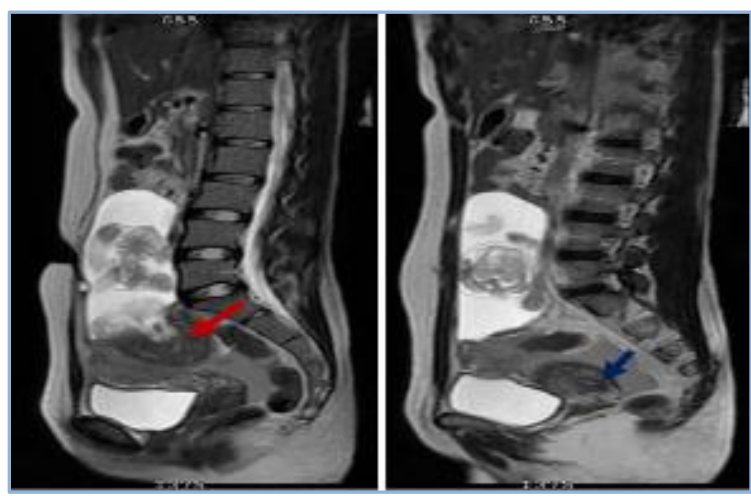

Figure 5 
Fig. (6): Showing Operative findings.

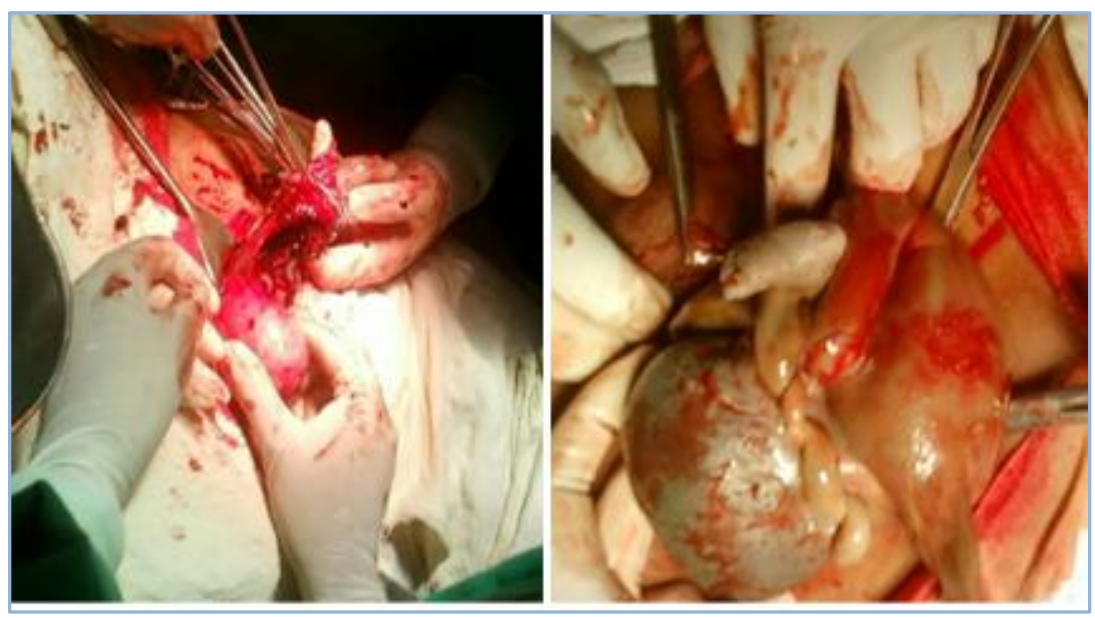

Figure 6

OPERATIVE FINDINGS: Explorative laparotomy shows a unicornuate uterus (U) with rupture of Non-communicating rudimentary horn (RH) was confirmed and a dead fetus (F) was found in peritoneal cavity with minimal haemoperitoneum. Both the ovaries and tubes were normal Placenta was found separated in abdominal cavity. Excision of rudimentary horn, ipsilateral salpingectomy, and peritoneal toileting was done.

DISCUSSION: Rudimentary horn with a unicornuate uterus results from failure of complete development of one of the mullerian ducts and incomplete fusion with the contralateral side. In 83\% of cases the rudimentary horn is non-communicating.[4] Pregnancy in a non-communicating rudimentary horn occurs through transperitoneal migration of sperm or fertilized ovum.[5] It is associated with a high rate of spontaneous abortion, preterm labour, intrauterine growth retardation, intraperitoneal hemorrhage and uterine rupture.[6] Diagnosis prior to rupture is unusual, but could be made with ultrasonography and MRI. Tsafrir et al outlined a set of criteria for diagnosing pregnancy in the rudimentary horn. [7] They are: (1) A pseudo pattern of asymmetrical bicornuate uterus; (2) Absent visual continuity tissue surrounding the gestation sac and the uterine cervix. (3) Presence of myometrial tissue surrounding the gestation sac. None-the-less most cases remain undiagnosed until it ruptures and presents as an emergency. The usual outcome of rudimentary horn pregnancy is rupture in second trimester in $90 \%$ of cases with fetal demise.[8] however cases of pregnancy progressing to the third trimester and resulting in a live birth after caesarean section has been documented.[6] Pregnancy in rudimentary horn may not be as rare as previously calculated. In exhaustive review of the $20^{\text {th }}$ century literature, Nahum summarized 588 such cases. ${ }^{[9]}$ In that series, uterine rupture occurred in $80 \%$ of RHPs. Among these neonatal survival was only $6 \%$. The maternal mortality was $5.1 \%$, although none was reported after 1960. Cases of late and false diagnosis leading to uterine rupture have been reported repeatedly in the recent literature. In our case the diagnosis of RHP was challenging. Intra uterine pregnancy in a bicornuate uterus, ectopic pregnancy like tubal pregnancy, Cornual pregnancy and abdominal pregnancies are common sonographic differential diagnosis. The continuity between the endometrium lining the gestational sac and the other uterine horn is typical of pregnancy in a bicornuate uterus. 
Ectopic pregnancies beyond 12 weeks of gestation are rarely tubal. In cornual pregnancy, sonography will reveal an interstitial line that extends from the uterine cavity to the cornual gestational sac. The unicornuate uterus is a result of abnormal or failed development of one of the paired müllerian ducts. This group of anomalies can be further subdivided into 4 variants according to the American Fertility Society. The isolated unicornuate uterus is the most common, with a reported frequency of $35 \%$. When a rudimentary horn is present, it is noncavitary in $33 \%$ of cases, cavitary non-communicating in $22 \%$, and cavitary communicating in $10 \%$ a rudimentary horn is found in $84 \%$ unicornuate uterus. In its simplest form of an isolated unicornuate uterus, most patients remain asymptomatic and therefore go undetected unless the discovery is made as an incidental finding.

Patients who have a unicornuate uterus with a rudimentary horn have an increased incidence of gynecologic problems and tend to present at menarche or later in life with symptoms consisting of dysmenorrhea, chronic pelvic pain, hematometra, and hematosalpinx most commonly related to endometriosis. Finally, a third subset of patients will consist of women who present with pregnancyrelated complications such as first and second trimester abortions, preterm deliveries, intrauterine fetal demise, and ectopic pregnancies. A pregnancy can develop in both communicating and noncommunicating rudimentary horns. In the later type, it occurs by transperitoneal migration of sperm. This condition is undesirable because it nearly always results in rupture of the rudimentary horn, which may cause life threatening bleeding.

Rudimentary horn pregnancies as well as ectopic tubal and cornual pregnancies can occur in both communicating and non-communicating horns. In his review of 588 rudimentary horn pregnancies, Nahum found that $83 \%$ occurred in a non-communicating horn and that uterine rupture occurred in $90 \%$ of the cases in the second trimester.

CONCLUSION: There is a need of detailed clinical evaluation, increased awareness of this rare condition and high index of suspicion for early pre rupture diagnosis. Pregnancy in a noncommunicating rudimentary horn is extremely rare and usually terminates in rupture during first or second trimester of pregnancy. It can be missed in routine ultrasound scan and in majority of cases it is detected after rupture. It requires a high index of suspicion. If ultrasound shows abnormal relationship among the fetus, placenta, amniotic fluid and uterus and mal presentation especially transverse lie suspect ruptured RHP. MRI was performed after inconclusive ultrasound observations for the preoperative planning as well as to evaluate the myometrium surrounding the gestational sac and the placental location. MRI confirmed the diagnosis of RHP.

\section{REFERENCES:}

1. Tufail A, Hasmi HA: Ruptured ectopic pregnancy in rudimentary horn of the uterus. J Coll Physicians Surg Pak 2007, 17:105-106.

2. Ural SH, Artal R: Third trimester rudimentary horn pregnancy. A case report. J Reprod Med 1998, 37:919-921.

3. Nahum G: Rudimentary uterine horn pregnancy: case report on surviving twins delivered 8 days apart. J Reprod Med 1997, 42:525-532.

4. PK: Unicornuate uterus and rudimentary horn. Fertil steril 1997, 68:224-230.

5. Panayotidis C, Abdel-Fattah M, Leggott M: Rupture of rudimentary horn of a unicornuate Uterus at 15 weeks gestation. J Obstet Gynaecol 2004, 24:323-324. 


\section{CASE REPORT}

6. Jin Woo Shin, Hai Joong Kim: Case of live birth in a non-communicating rudimentary horn Pregnancy. J Obstet Gynaecol Res 2005, 31:329-331.

7. Tsafrir A, Rojansky N, Sela HY, et al.: Rudimentary horn pregnancy: first trimester pre-rupture sonographic diagnosis and confirmation by magnetic resonance imaging. J Ultrasound Med 2005, 24:219-223.

8. Liu MM: Unicornuate uterus with rudimentary horn. Int J Gynaecol Obstet 1994, 44:149-153.

9. Nahum, G.G., 2002. Rudimentary uterine horn pregnancy. The 20th century worldwide experience of 588 cases. J. Reprod. Med.

\section{AUTHORS:}

1. B. Anuradha

2. B. Sudharani

3. K. Sambasiva

4. V. Sarath Chand

\section{PARTICULARS OF CONTRIBUTORS:}

1. Associate Professor, Department of Radiology, Rangaraya Medical College, Kakinada.

2. Assistant Professor, Department of Radiology, Rangaraya Medical College, Kakinada.

3. Assistant Professor, Department of Radiology, Rangaraya Medical College, Kakinada.

FINANCIAL OR OTHER COMPETING INTERESTS: None
4. Assistant Professor, Department of Radiology, Rangaraya Medical College, Kakinada.

\section{NAME ADDRESS EMAIL ID OF THE CORRESPONDING AUTHOR:}

Dr. B. Anuradha, Associate Professor and Incharge HOD, Department of Radiology, Government General Hospital, Kakinada, East Godavari District, Andhra Pradesh. E-mail: anuradhabonthu@yahoo.co.in

Date of Submission: 03/07/2015.

Date of Peer Review: 04/07/2015.

Date of Acceptance: 23/07/2015.

Date of Publishing: 17/08/2015. 